\title{
A combinatorial description of the Heegaard Floer contact invariant
}

\author{
Olga Plamenevskaya
}

\begin{abstract}
We observe that the Heegaard Floer contact invariant is combinatorial by applying the algorithm of Sarkar-Wang to the description of the contact invariant due to Honda-Kazez-Matić. We include an example of this combinatorial calculation.
\end{abstract}

57R17; 57R58

\section{Introduction}

Recent months have seen a significant advance in Heegaard Floer theory: it turned out that certain Heegaard Floer homologies admit a purely combinatorial description. In particular, it was shown by Manolescu, Ozsváth and Sarkar [8] that the Heegaard Floer homologies of a knot can be computed from a grid diagram of the knot by a simple combinatorial procedure. Heegaard Floer homology $\widehat{H F}(Y)$ of a 3-manifold $Y$ also admits a combinatorial description (see Sarkar and Wang [11]) but this description is less straightforward. Starting from an arbitrary admissible Heegaard diagram of $Y$, one has to change the $\beta$ curves by isotopies and handleslides so that in the resulting Heegaard diagram almost all the domains of holomorphic disks are squares or bigons. It is then easy to understand the moduli spaces of the holomorphic disks needed to compute the differential, since squares and bigons with Maslov index $=1$ admit a unique (up to an $\mathbb{R}$-action) holomorphic representative. (We assume that the reader is familiar with the basic setup of the Heegaard Floer theory; see Ozsváth and Szabó [10] for a survey.)

For a contact 3-manifold $(Y, \xi)$, Ozsváth and Szabó introduce in [9] an invariant $c(\xi)$ which is a distinguished element of $\widehat{H F}(-Y)$ (defined up to sign for the theory with $\mathbb{Z}$ coefficients). Since non-vanishing of $c(\xi)$ implies that $\xi$ is tight, this invariant gives a powerful tool for establishing the tightness of a contact structure (see Lisca and Stipsicz $[5 ; 6 ; 7])$.

The invariant $c(\xi)$ is defined in [9] via an open book decomposition of $(Y, \xi)$, as follows. If the genus of the page of the open book is $g>1$ (and the binding is connected), we consider a certain Heegaard diagram for $Y$ of genus $2 g+1$, compatible with 
the open book. The generators of the group $\widehat{C F}(-Y)$ are given by $(2 g+1)$-tuples of the intersection points of $\alpha$ - and $\beta$-curves in the diagram, and a distinguished $(2 g+1)$-tuple gives a cycle $\mathbf{c}$ which descends to the invariant $c(\xi)$ in homology.

Our goal is to show that $c(\xi)$ can be computed in a combinatorial fashion. We would like to apply the Sarkar-Wang algorithm [11] to obtain a Heegaard diagram where the holomorphic disks can be easily identified. However, we are concerned with a specific cycle, not the homology group as a whole, and the isotopies and/or handleslides of the $\beta$-curves performed on the Heegaard surface would possibly affect the generator c. Indeed, the homology class of a geometric generator $\mathbf{c}$ can change even if all the isotopies are supported away from the intersection points forming $\mathbf{c} ; \mathbf{c}$ might even no longer be a cycle after the isotopy. Consider for example the genus 1 Heegaard diagram for $S^{1}$ given by an $\alpha$ - and a $\beta$-curve on the torus intersecting at one point. This intersection point $\mathbf{x}$ is a cycle which generates $\widehat{H F}\left(S^{3}\right)=\mathbb{Z}$. We isotope the $\beta$-curve to introduce two extra intersection points, $\mathbf{y}$ and $\mathbf{z}$, as shown on Figure 1. Now, we have $\partial \mathbf{x}=\mathbf{y}, \partial \mathbf{z}=\mathbf{y}$ in the chain complex $\widehat{C F}\left(S^{3}\right)$ for the new Heegaard diagram; so $\mathbf{x}$ is no longer a cycle, and $\widehat{H F}\left(S^{3}\right)$ is generated by $\mathbf{x}-\mathbf{z}$.
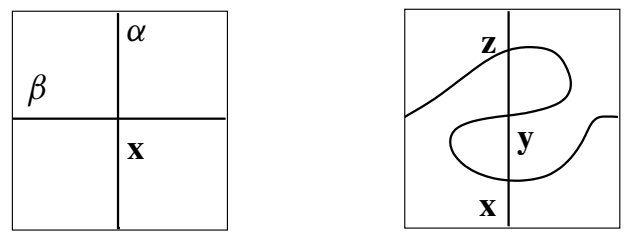

Figure 1: The Heegaard surface here is a torus obtained by identifying the opposite sides of the square. After the isotopy of the $\beta$-curve, $\mathbf{x}$ is no longer a cycle in the Heegaard Floer complex.

This shows that we need to be more careful. Fortunately, there is an alternate geometric description of $c(\xi)$ due to Honda-Kazez-Matić: in [3] they show that $c(\xi)$ can be found from a Heegaard diagram which is different from and somewhat simpler than the one in Ozsváth and Szabó [9]. This alternative Heegaard diagram is easier to handle, and fits well with the Sarkar-Wang algorithm.

We combine the arguments from [3] and [11] to establish our main result in the next section.

Remark One could in principle extract information about $c(\xi)$ in a combinatorial way from knot Floer homology. When the binding $K$ of the open book for $(Y, \xi)$ is connected, $c(\xi)$ is the image in $\widehat{H F}(-Y)$ of the generator of the knot homology 
$\widehat{H F K}(-Y, K, S,-g)$. (Here $S$ stands for the page of the open book; since $K$ is fibered, $\widehat{H F K}(-Y, K, S,-g)=\mathbb{Z}$ by Ozsváth and Szabó [9]. We consider the image of the generator under the spectral sequence from $\widehat{H F K}(-Y, K)$ to $\widehat{H F}(-Y)$.) Using the Sarkar-Wang algorithm for knots, we could find a Heegaard diagram for $(-Y, K)$ yielding a combinatorial description of the knot chain complex. Pinpointing the cycle corresponding to the generator of $\widehat{H F K}(-Y, K, S,-g)$, we could then determine $c(\xi)$. However, this procedure is not very explicit, and would be hard to perform in practice.

\section{The main result}

We first recall the construction from Honda, Kazez and Matić [3]. Let $(S, h)$ be an open book decomposition for the contact manifold $(Y, \xi)$; here $S$ denotes the page of the open book, and $h$ the monodromy. This means that $Y$ is homeomorphic to $S \times[0,1] / \sim$, where the equivalence relation $\sim$ is given by

$$
\begin{aligned}
(x, 1) & \sim(h(x), 0), & & x \in S \\
(x, t) & \sim\left(x, t^{\prime}\right), & & x \in \partial S, \quad t, t^{\prime} \in[0,1] .
\end{aligned}
$$

The open book produces a Heegaard splitting $Y=H_{1} \cup H_{2}$, with $H_{1}=S \times[0,1 / 2] / \sim$, $H_{2}=S \times[1 / 2,1] / \sim$. The Heegaard diagram for $Y$ can then be given by the Heegaard surface $\Sigma=S_{1 / 2} \cup-S_{0}$, and the $\alpha$ - and $\beta$-curves, defined as follows. Consider a set of disjoint, properly embedded $\operatorname{arcs}\left\{a_{1}, a_{2}, \ldots, a_{n}\right\}$ on $S$ such that $S \backslash \bigcup a_{i}$ is a single polygon. Obtain $\operatorname{arcs} b_{i}$ by changing the $\operatorname{arcs} a_{i}$ via a small isotopy so that the endpoints of $a_{i}$ are isotopied along $\partial S$ (in the direction dictated by the boundary orientation), the $\operatorname{arcs} a_{i}$ and $b_{i}$ intersect transversely at one point, and the sign of this intersection is positive (the orientation of $b_{i}$ is induced from the orientation of $a_{i}$ by the isotopy). The curves $\alpha_{i}=\partial\left(a_{i} \times[0,1 / 2]\right)$ and $\beta_{i}=\partial\left(b_{i} \times[1 / 2,1]\right)$ form attaching circles for the handlebodies $H_{1}$ and $H_{2}$; they can be thought of as the $\alpha-$ and $\beta-$ curves on $\Sigma$. We can write

$$
\alpha_{i}=a_{i} \times\{1 / 2\} \cup a_{i} \times\{0\}, \quad \beta_{i}=b_{i} \times\{1 / 2\} \cup h\left(b_{i}\right) \times\{0\} ;
$$

thus, the intersection of $\alpha$ - and $\beta$-curves with $S_{1 / 2}$ is completely standard (and given by $\left.a_{i}, b_{i}\right)$; the picture on $S_{0}$ depends on the monodromy $h$. For an illustration of such Heegaard diagram, see [3], or look at the example in the next section. The basepoint $z_{0}$ is placed on $S_{1 / 2}$ in the polygonal region (not in the thin strips between $a_{i}$ 's and $b_{i}$ 's); we denote this polygonal region by $D_{0}$. Now, let $c_{i}$ be the intersection point between $a_{i}$ and $b_{i}$ on $S_{1 / 2}$. It is shown in [3] that the element $\mathbf{c}=\left(c_{1}, c_{2}, \ldots, c_{n}\right) \in$ $\widehat{C F}\left(\Sigma, \beta, \alpha, z_{0}\right)$ is a cycle which descends to the element $c(\xi)$ in the homology $H_{*}\left(\widehat{C F}\left(\Sigma, \beta, \alpha, z_{0}\right)\right)=\widehat{H F}(-Y)$. 
(Note that the roles of the $\alpha$ - and $\beta$-curves are interchanged because we need the homology of $-Y$ instead of the homology of $Y$. So for a Heegaard diagram of genus $g$, a Whitney disk from $\mathbf{x}$ to $\mathbf{y}$ is now a map $\phi: \mathbb{D} \rightarrow \operatorname{Sym}^{g} \Sigma$ such that $\phi(i)=\mathbf{x}$, $\phi(-i)=\mathbf{y}$, and $\mathbb{D} \cap\{\operatorname{Re} z>0\}$ is mapped into $\mathbb{T}_{\alpha}=\alpha_{1} \times \cdots \times \alpha_{g}$, while $\mathbb{D} \cap\{\operatorname{Re} z<0\}$ is mapped into $\mathbb{T}_{\beta}=\beta_{1} \times \cdots \times \beta_{g}$. This does not affect the combinatorial algorithm that we will be using later.)

Applying the idea of Sarkar-Wang, we would like to find an open book decomposition for $Y$ such that all but one region in the corresponding Heegaard diagram are bigons or squares (here and below, a region is a connected component in the complement of $\alpha$ - and $\beta$-curves in $\Sigma$ ).

Theorem 2.1 There exists an open book $\left(S, h^{\prime}\right)$ for $(Y, \xi)$, such that the Heegaard diagram described above has only bigon and square regions (except for the polygonal region $D_{0} \subset S_{1 / 2}$ ). The monodromy $h^{\prime}$ differs from the monodromy of the given open book $(S, h)$ by an isotopy, i.e. $h^{\prime}=\phi \circ h$, where $\phi: S \rightarrow S$ is a diffeomorphism fixing the boundary and isotopic to identity.

Proof The algorithm of Sarkar and Wang [11] tells us to get rid of non-disk regions and $2 n$-gons with $n>2$ by performing isotopies (finger moves) of $\beta$-curves. First, the non-disks are killed; then, the $2 n$-gons are dealt with (one after another) roughly as follows. We look at the "distance" between a given region $D$ and the region $D_{0}$ (the minimal number of intersections between the $\beta$-curves and an arc connecting $z_{0}$ to an interior point of $D$ ), and number all the regions $D_{0}, D_{1}, D_{2}$, etc, so that the distance between $D_{m}$ and $D_{0}$ increases with $m$. Sarkar and Wang explain how to perform finger moves that break up a given $2 n-$ gon $D_{k}$ into polygons with fewer sides, pushing part of the boundary of $2 n$-gon into other regions (typically labelled with smaller numbers). During this process, the regions $D_{l}$ with $l>k$ which are already bigons or squares remain bigons or squares, so the process eventually terminates.

We observe that for our Heegaard diagram coming from an open book, all the finger moves can be performed in the $S_{0}$ part of $\Sigma$. Indeed, every boundary curve of every region in $\Sigma$ intersects $S_{0}$, and moreover, any two edges of a given region can be connected by an arc contained in $S_{0}$, so we can perform finger moves along such arcs.

(Note that our Heegaard diagram is weakly admissible from the start by Honda, Kazez and Matić [3].)

Apart from the finger moves, handleslides of the $\beta$-curves are sometimes needed in the algorithm of [11]. After such a handleslide, the Heegaard diagram would no longer be compatible with considerations in [3]. However, it turns out that the need for 
handleslides fortunately does not arise in our case. Indeed, a handleslide in [11] is only needed when a finger is pushed through a collection of adjacent regions, none of which has a smaller distance from $D_{0}$ (in particular, none of these regions is $D_{0}$ ), and then comes back to the region where it started. This means that the finger goes around a full copy of some curve $\beta_{i}$. Because each of the $\beta$-curves forms part of the boundary of $D_{0}$, and $a_{i}$ and $b_{i}$ intersect at one point in $S_{1 / 2}$, it follows that the finger has to go through $D_{0}$, which is a contradiction.

Therefore, we can obtain a "nice" Heegaard diagram (in the terminology of [11]) by performing a sequence of isotopies on $S_{0} \subset \Sigma$ (away from boundary). A composition of these isotopies gives a diffeomorphism $\phi$ and the open book $(S, \phi \circ h)$ equivalent to the open book $(S, h)$ we started with.

Remark Using an open book decomposition as above, we can compute the Heegaard Floer homology of an arbitrary 3-manifold combinatorially via an algorithm that uses isotopies of $\beta$-curves only, and no handleslides. (This strengthens slightly the algorithm of [11].)

To complete the proof we refer to the following lemma.

Lemma 2.2 (Sarkar and Wang [11, Theorem 1.1]) For a nice Heegaard diagram, all the differentials in the "hat" Heegaard Floer complex can be computed combinatorially.

We recall the structure of these differentials from [11] as we'll need it to compute an example. Stabilizing the open book if necessary, we can assume that the genus of our Heegaard diagram is at least 2. Suppose we look for holomorphic representatives of a Whitney disk $\phi \in \pi_{2}(\mathbf{x}, \mathbf{y})$ connecting the points $\mathbf{x}$ and $\mathbf{y}$ and missing the marked point $z$. The domain of $\phi$ is then a linear combination of some regions $D_{i}, D=\sum_{i=1}^{k} a_{i} D_{i}$. If $\phi$ has holomorphic representatives, we must have $a_{i} \geq 0$. Lipshitz's formula [4] for the Maslov index implies that when $\mu(\phi)=1, D$ must be an embedded square (tiled by squares), or an embedded bigon (tiled by squares and a bigon). The domain $D$ must also be empty, in the sense that it cannot contain any $x$-coordinate in its interior. Assuming that these conditions hold, the transversality condition is also satisfied (for a generic perturbation of $\alpha$-and $\beta$-curves), and $\phi$ has a unique holomorphic representative (up to an $\mathbb{R}$-action). Whitney disks that are not empty embedded squares or bigons do not contribute to the differential.

Remark To find out whether the invariant $c(\xi)$ vanishes or not, we only need to understand the moduli spaces for the disks contributing to the differentials possibly killing the element $\mathbf{c}$ (but not other disks in the picture). However, the domain for such 
a holomorphic disk may contain more than one region, so we still need to simplify all regions in the Heegaard diagram (not only those passing through the "thin strips" on $\left.S_{1 / 2} \subset \Sigma\right)$.

\section{An example}

In this section we illustrate the combinatorial calculation of $c(\xi)$ by the following example. (We consider $\mathbb{Z} / 2$ coefficients for simplicity, and only show that $c(\xi)$ is non-zero without computing $\widehat{H F}(-Y))$. Consider the contact manifold $(Y, \xi)$ given by the open book whose page is a four-punctured sphere, and the monodromy is the composition of two positive Dehn twists around the curves $\gamma_{1}$ and $\gamma_{2}$ shown in Figure 2. Of course, this contact manifold is easy to understand without Heegaard Floer theory: by Giroux [2], $\xi$ is Stein fillable (and therefore tight); moreover, we can perform two positive destabilizations to see that $\xi$ is in fact simply the standard tight contact structure on $S^{1} \times S^{2}$ (given by the open book with an annular page and trivial monodromy).

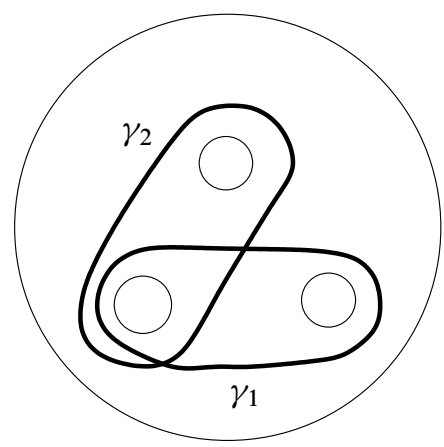

Figure 2: The monodromy of the open book is given by a positive Dehn twist around $\gamma_{1}$ followed by a Dehn twist around $\gamma_{2}$.

Our point, however, is to illustrate how Theorem 2.1 works for this open book. First, we look at the Honda-Kazez-Matić-style Heegaard diagram for $(Y, \xi)$ (Figure 3). (We draw the picture by applying the two Dehn twists directly; the use of the lantern relation - see Dehn [1] - can somewhat simplify the task.) Observe that there are two "bad" regions, both hexagons, in the complement of $\alpha$ - and $\beta$-curves on the Heegaard surface.

We get rid of them by applying the Sarkar-Wang algorithm as described in Theorem 2.1 , which in this case amounts simply to winding two of the $\beta$-curves as shown in 


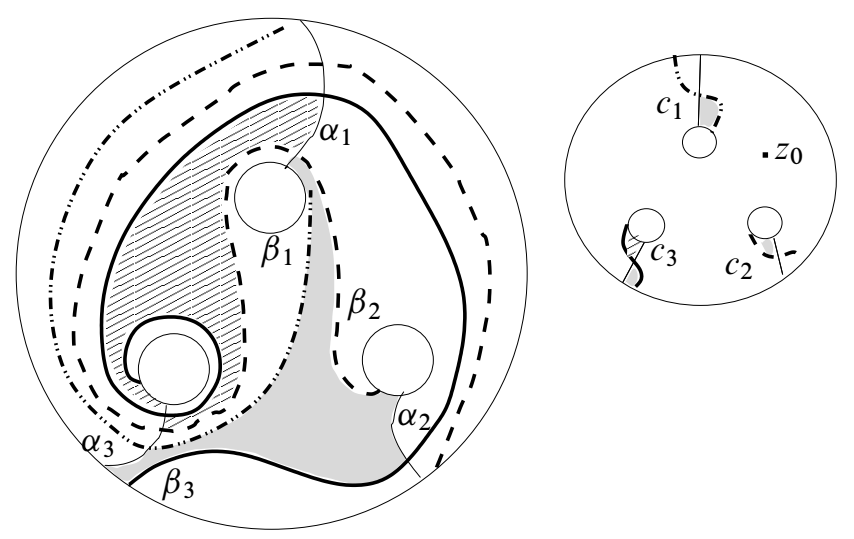

Figure 3: This is a Heegaard diagram for $(Y, \xi)$. The surfaces $S_{0}$ and $S_{1 / 2}$, the "bottom" and "top" parts of $\Sigma$, are shown separately $\left(S_{1 / 2}\right.$ is smaller because it's completely standard). Note that $\Sigma=-S_{0} \cup S_{1 / 2}$; the picture shows $S_{0}$, not $-S_{0}$. The thin curves are the $\alpha$-curves, the thicker lines (solid, dashed and dash-dotted) are the $\beta$-curves. The contact element $\mathbf{c}=\left(c_{1}, c_{2}, c_{3}\right)$ lies on $S_{1 / 2}$. The "bad" regions on $\Sigma$ are shown.
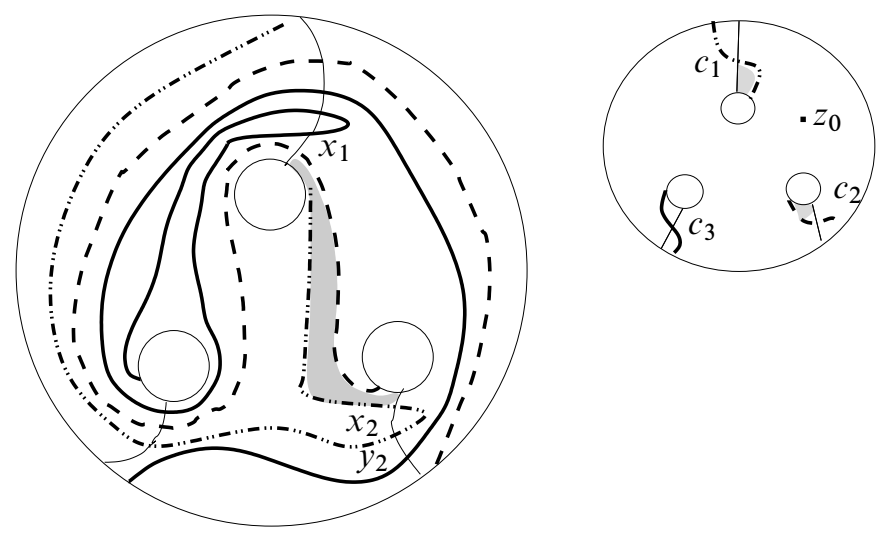

Figure 4: The Heegaard diagram after winding. The domain of the holomorphic disk connecting $\mathbf{x}=\left(x_{1}, x_{2}, c_{3}\right)$ and $\mathbf{c}=\left(c_{1}, c_{2}, c_{3}\right)$ is shaded.

Figure 4: as explained in the end of section 2, we just have to push fingers out of bad regions into $D_{0}$.

We obtain a Heegaard diagram shown on Figure 4. Examining it, we see that there is only one possible domain of a differential going to $\mathbf{c}$ from another point $\mathbf{x}$; the point 
$\mathbf{x}=\left(x_{1}, x_{2}, c_{3}\right)$ is shown in the picture, and the domain is shaded. (Recall that the intersection of all such domains with $S_{1 / 2}$ should lie in the thin strips between $a_{i}$ and $b_{i}$, since the domain must not contain $z_{0}$.) But then we have $d \mathbf{x}=\mathbf{c}+\mathbf{y}$, where $\mathbf{y}=\left(x_{1}, y_{2}, c_{3}\right)$, since there is a bigon connecting $\mathbf{x}$ and $\mathbf{y}$ (and no other Whitney disks from $\mathbf{x}$ ). This shows that $\mathbf{c}$ is not a boundary, and so $c(\xi) \neq 0$. We can conclude that the contact structure $\xi$ is tight.

Remark It would be interesting to use the combinatorial approach to investigate some of the contact structures from Lisca and Stipsicz [5; 6; 7], and especially contact structures that can't be handled by those methods. In particular, certain planar open books arising from contact surgeries may produce diagrams that are not too hard to analyze. We hope to return to this in a future paper.

\section{References}

[1] M Dehn, Papers on group theory and topology, Springer, New York (1987) MR881797

[2] E Giroux, Géométrie de contact: de la dimension trois vers les dimensions supérieures, from: "Proceedings of the International Congress of Mathematicians, Vol. II (Beijing, 2002)", Higher Ed. Press, Beijing (2002) 405-414 MR1957051

[3] K Honda, W Kazez, G Matić, On the contact class in Heegaard Floer homology arXiv:math.GT/0609734

[4] R Lipshitz, A cylindrical reformulation of Heegaard Floer homology, Geom. Topol. 10 (2006) 955-1097 MR2240908

[5] P Lisca, A I Stipsicz, Ozsváth-Szabó invariants and tight contact three-manifolds I, Geom. Topol. 8 (2004) 925-945 MR2087073

[6] P Lisca, A I Stipsicz, Ozsváth-Szabó invariants and tight contact three-manifolds II, J. Differential Geom. 75 (2007) 109-141

[7] P Lisca, A I Stipsicz, Ozsváth-Szabó invariants and tight contact three-manifolds III arXiv:math.SG/0505493

[8] C Manolescu, P Ozsváth, S Sarkar, On combinatorial link Floer homology arXiv : math.GT/0610559

[9] P Ozsváth, Z Szabó, Heegaard Floer homology and contact structures, Duke Math. J. 129 (2005) 39-61 MR2153455

[10] P Ozsváth, Z Szabó, Heegaard diagrams and Floer homology, from: "International Congress of Mathematicians Vol. II”, Eur. Math. Soc., Zürich (2006) 1083-1099 MR2275636

[11] S Sarkar, J Wang, An algorithm for computing some Heegaard Floer homologies arXiv:math.GT/0607777 
Department of Mathematics, SUNY Stony Brook Stony Brook NY 11794, USA

olga@math.sunysb.edu

Received: 28 May 2007 